\title{
Cirurgia segura: a enfermagem protagonizando a segurança do paciente no Centro Cirúrgico
}

\author{
Safe Surgery: Nursing Leading the Patient Safety at the Surgical Center \\ Cirugía segura: enfermería que llevalaseguridaddel paciente enel centro quirúrgico
}

\author{
Wanderson Alves Ribeiro ${ }^{1 *}$, Iago de Freitas Mattos $^{2}$, Maicon Costa de Morais ${ }^{3}$, Douglas Mendes da \\ Silva Souza ${ }^{4}$, Carla de Souza Couto ${ }^{5}$, Leandro Mendes Martins ${ }^{6}$
}

Como citar esse artigo. Ribeiro, WA; Mattos, IF; de Morais, MC; Souza, DMS; Couto, CS; Martins, LM. Cirurgia segura: a enfermagem protagonizando a segurança do paciente no Centro Cirúrgico. Revista Pró-UniverSUS. 2019 Jan./Jun.; 10 (1): 66-71

\section{Resumo}

Estudo de pesquisa bibliográfica com abordagem qualitativa que tem como objetivo descrever a relevância do procedimento cirúrgico seguro e evidenciar as implicações dos enfermeiros e sua equipe no processo da cirurgia segura. Desta forma, salientando a realidade atual dos hospitais, que devem seguir todo o protocolo que foi estabelecido, visando o bem estar físico e emocional do paciente, desde o momento de sua admissão até a alta hospitalar. Os dados foram coletados mediante a uma serie de estudos e pesquisas em artigos científicos e sites. Por sua vez ajudando a refletir como o cuidado produzido repercute não apenas sobre a pessoa, mas também sobre a instituição e os profissionais que executam de maneira correta os passos do manual de cirurgia segura.

Palavras-chave: Cirurgia, Cirurgia segura, Enfermagem.

\begin{abstract}
A qualitative study of a bibliographic research that aims to describe the relevance of the safe surgical procedure and to highlight the implications of nurses and their team in the process of safe surgery. In this way, highlighting the current reality of hospitals, which must follow all the protocol that was established, aiming at the patient's physical and emotional well-being, from the moment of admission to discharge. The data were collected through a series of studies and research in scientific articles and websites. In turn helping to reflect how the care produced impacts not only on the person, but also on the institution and the professionals who perform correctly the steps of the safe surgery manual.

Keywords: Surgery, Safe surgery, Nursing.
\end{abstract}

\section{Resumen}

Estudio de investigación bibliográfica con abordaje cualitativo que tiene como objetivo describir la relevancia del procedimiento quirúrgico seguro y evidenciar las implicaciones de los enfermeros y su equipo en el proceso de la cirugía segura. De esta forma, subrayando la realidad actual de los hospitales, que deben seguir todo el protocolo que fue establecido, visando el bienestar físico y emocional del paciente, desde el momento de su admisión hasta el alta hospitalaria. Los datos fueron recolectados mediante una serie de estudios e investigaciones en artículos científicos y sitios. Por su parte, ayudando a reflexionar cómo el cuidado producido repercute no sólo sobre la persona, sino también sobre la institución y los profesionales que ejecutan de manera correcta los pasos del manual de cirugía segura.

Palabras clave: Cirugía, Cirugía segura, Enfermería.

Afiliação dos autores:1Enfermeiro. Mestrando Acadêmico em Ciências do Cuidado em Saúde, UFF. RJ, Brasil. Email: nursing_war@hotmail.com ORCID: https://orcid.org/00000001-8655-3789

2Enfermeiro. Pós-Graduado em Enfermagem em Centro Cirúrgico FAVENI, Brasil. E-mail: soares_mattos@hotmail.com ORCID: ORCID: https://orcid.org/0000-0001-5489-7931 RJ, Brasil. Email: brunaporath@gmail.com ORCID: https://orcid.org/0000-0002-1400-4147

3Enfermeiro. Pós-Graduando em CTI Pediátrico e Neonatologia, FAVENI, Brasil. Email: maiconenf2406@gmail.com ORCID: https://orcid.org/0000-0002-5450-7574

4Enfermeiro. Enfermeiro. Pós em Gestão em Estratégia Saúde da Família, FAVENI, Brasil. Email: douglaska souza@globo.com ORCID: https://orcid.org/0000-0002-4283-7701

5Enfermeira. Docente Titular do Curso de Formação Técnica em Enfermagem do CTEF. Email: desouza4_@hotmail.com ORCID: https://orcid.org/0000-0003-3999-0753

6 Enfermeiro. Coordenador. Docente Titular Curso de Formação Técnica em Enfermagem, CTEF. Email: leandro.mendes.martins@hotmail.com ORCID: https://orcid.org/0000-0003 -0903-877X

* Email de correspondencia: nursing_war@hotmail.com.

Recebido em: 12/12/18. Aceito em: 25/05/19. 


\section{Introdução}

A etimologia do termo cirurgia remonta do vocábulo latim, que por sua vez, tem origem grega, também chamada de procedimento cirúrgico. Ocorre no ato em que o cirurgião realiza uma intervenção seja ela manual ou instrumental no corpo do paciente para diagnosticar ou tratar as doenças, melhorando desta forma a funcionalidade ou aparência do corpo. ${ }^{1}$

Existem diversos ramos dentro da cirurgia, que vão se encarregar de cada região ou órgão do corpo, tendo objetivos e causas diferentes. Desta forma são classificadas como: Cirurgia geral, torácica, plástica, ortopédica, vascular, cardíaca e entre outras. Existindo para realização de cada uma, a equipe cirúrgica que será composta por: Cirurgião chefe, cirurgião assistente, anestesista, instrumentador cirúrgico e o circulante de sala. $^{2}$

O centro cirúrgico é o local onde ocorre o ato operatório, sendo uma área complexa, de acesso restrito, possuindo particularidades na sua estrutura física e atendendo as normatizações da Agência Nacional de Vigilância Sanitária. Ele devera conter ambientes de apoio como sala para guardar equipamentos, vestiários com banheiro, sala administrativa, laboratório, copa, sala de espera para os acompanhantes com banheiro, sala de estar para os funcionários, área destinada às cadeiras de rodas e macas, entre outros. ${ }^{3}$

Deve ser um local de fácil acesso a outros setores do hospital, como os de: Internação, Centro de Terapia Intensiva, Pronto Socorro, Central de Material e Esterilização, Raios-X, Laboratório, Lavanderia e Farmácia. Evidenciando que é de obrigação da instituição seguir o protocolo de recomendações gerais para as instalações da área. ${ }^{3}$

A estrutura física do Centro Cirúrgico será dividida de acordo com seus riscos, sendo classificadas em: Não restrita: as áreas de circulação livre (vestiários, corredor de entrada e sala de espera de acompanhantes), semi-restritas: pode haver circulação tanto do pessoal como de equipamentos, sem provocarem interferência nas rotinas de controle e manutenção da assepsia (salas de material, administrativa, copa e expurgo), e por fim, a área restrita: o corredor interno, as áreas de escovação das mãos e a sala de operação (SO) (para evitar infecção operatória, limita-se a circulação de pessoas, equipamentos e materiais).; ${ }^{2 ; 3}$

Sendo desta forma seu principal objetivo é atender da melhor maneira possível o paciente que será submetido a um procedimento cirúrgico, seja ele eletivo, de urgência, ou emergencial, propiciando à equipe cirúrgica todas as condições de atendimento e ao paciente a certeza de um procedimento seguro. Tendo como finalidade a realização de intervenções cirúrgicas e de devolver o paciente à sua unidade de origem na melhor condição possível de integridade. ${ }^{2 ; 3}$

$\mathrm{O}$ processo cirúrgico se inicia a partir do pré-operatório mediato no qual é diagnosticada a necessidade de ocorrer o ato operatório até vinte quatro horas antes da cirurgia, passando para o pré-operatório imediato, que é correspondente as vinte quatro horas que antecedem o procedimento, no qual são realizados todos os cuidados necessários para que não ocorram diversas complicações. ${ }^{4}$

Apartir desse momento entra-se no intra operatório no qual o paciente é transferido para o bloco cirúrgico até sua admissão na unidade de cuidado pós-anestésica. Nesse momento ocorre a fase pós-operatória que é dividido em três momentos: Pós-operatório imediato (24 horas pós-cirurgia), pós-operatório mediato (que são das 24 horas após a cirurgia até sete dias depois) e o pós-operatório tardio (após os sete dias, onde é reconhecida a alta). ${ }^{4}$

A cirurgia segura consiste em uma estratégia importante para tornar o procedimento cirúrgico mais seguro e ajudar a equipe de saúde a reduzir a possibilidade de ocorrência de danos ao paciente, promovendo a realização do procedimento certo, no local e paciente corretos. Mediante a esses fatores e seguindo as diretrizes da Organização Mundial de Saúde (OMS), estará garantindo ao cliente sua segurança. ${ }^{5}$

Sobre essa temática, temos como base a Portaria do MS 529/2013 que cria a Política Nacional de Segurança do Paciente, e o Manual da ANVISA "Cirurgias Seguras Salvam Vidas". Além desses documentos, temos o Manual do Conselho Regional de Enfermagem de São Paulo (COREN-SP), que aborda os 10 passos para a segurança do paciente, no qual o $4^{\circ}$ passo é Cirurgia Segura. $^{5}$

No decorrer desse processo torna-se essencial o papel da enfermagem, principalmente do enfermeiro lotado na unidade, que ira ficar responsável por realizar o check list, este baseado no quarto passo da cirurgia segura. Neste momento cabem também avaliar as condições físicas e psicologias, verificar o uso de prótese dentário e de diversos outros cuidados que deveram ser prestados ao admitir o paciente no Centro Cirúrgico. ${ }^{6}$

Vale ressaltar que a equipe de enfermagem que atua no Centro Cirúrgico é composta por pessoas de vários níveis, com responsabilidades diferentes. A quantidade desse pessoal varia conforme a complexidade e o volume de trabalho existente na Unidade, mas em geral a equipe é composta de: Enfermeiro, Técnico ou Auxiliar de Enfermagem. ${ }^{6}$

Diante da problemática apresentada podese destacar como objeto de estudo a importância da enfermagem no protocolo de cirurgia segura.

Para tal, traçou-se a seguinte questão norteadora: Qual a relevância da enfermagem no protocolo de cirurgia segura?

Diante disso, a pesquisa tem como objetivo 
descrever a relevância de enfermagem no protocolo de cirurgia segura e evidenciar os fatores que põem em risco a segurança do paciente cirúrgico.

\section{Metodologia}

Trata-se de um estudo do tipo de pesquisa bibliográfica com abordagem qualitativa. A pesquisa bibliográfica é a atividade de organizar, estruturar e realizar uma coleta de dados que irão sustentar todas as fases da pesquisa, uma vez que auxilia na definição do problema, na determinação dos objetivos, na construção de hipóteses e na justificativa da escolha do tema. ${ }^{7}$

A pesquisa qualitativa é indutiva, isto é, o pesquisadordesenvolveconceitos, ideiaseentendimentos a partir de padrões encontrados nos dados, focando no caráter subjetivo do objeto analisado, e assim estudando as suas particularidades e experiências individuais. ${ }^{8}$

Para o levantamento dos artigos, realizou-se uma busca na Biblioteca Virtual em Saúde BVS, a partir da base de dados: Literatura Latino-Americana e do Caribe em Ciências da Saúde (LILACS), Coleção Nacional das
Fontes de Informação do SUS (COLECIONA SUS) e Base De Dados de Enfermagem (BDENF). Utilizando os descritores: Cirurgia, cirurgia segura e enfermagem, com o operador booleano and a partir da sua confirmação nos Descritores em Ciência da Saúde (DECS).

Foram adotados como critério de inclusão: artigos relacionados à temática de estudo, da área de enfermagem, do idioma português, com o texto na integra e com recorte temporal de dez anos. Como critérios de exclusão definiu-se o descarte dos artigos sem relação temática com o estudo ou duplicidade de artigos.

Para realizar a busca dos artigos foram associados os descritores utilizando o operador booleano and, sendo utilizados todos os critérios de exclusão e inclusão para selecionar os artigos e refinar a pesquisa.

Após concluir a pesquisa foram selecionados 07 artigos, onde será realizada uma leitura crítica e análise dos mesmos, para que seja obtido o melhor resultado possível dentro do tema baseado.

Quadro 1. Artigos encontrados e selecionados nas bases de dados associando os descritores utilizando o operador booleanoand.

\begin{tabular}{|c|c|c|c|}
\hline Base de Dados & $\begin{array}{c}\text { Cirurgia segura and } \\
\text { Enfermagem }\end{array}$ & $\begin{array}{c}\text { Cirurgia and } \\
\text { Enfermagem }\end{array}$ & $\begin{array}{c}\text { Cinurgia Segura and } \\
\text { Cirurgia and } \\
\text { Enfermagem }\end{array}$ \\
\hline LILACS & 212 & 543 & 4 \\
\hline COLECIONA SUS & 13 & 45 & 2 \\
\hline BDENF & 122 & 195 & 1 \\
\hline
\end{tabular}

\section{Resultados}

$\mathrm{Na}$ análise foi observado que $53 \%$ dos artigos encontrados discutem sobre a importância da cirurgia segura, 30\% discutem sobre a relevância da enfermagem no Centro Cirúrgico, e 17\% discutem a aplicação dos enfermeiros no processo de cirurgia segura.

\section{A importância da cirurgia segura e os fatores determinantes para o paciente}

A cirurgia Segura contempla medidas adotadas para redução do risco de eventos (danos ao paciente) que podem acontecer antes, durante e após as cirurgias. $\mathrm{O}$ resultado esperado é mais segurança ao paciente com menos chance de erros assistenciais. A Organização Mundial da Saúde (OMS) instituiu a Aliança Mundial para a Segurança do Paciente, onde recomenda aos países, maior atenção à segurança do paciente. Assim foi desenvolvido um check list que deve ser aplicado a todos os pacientes cirúrgicos (TIME OUT).

O Time out é uma pausa antes da indução anestésica e incisão cirúrgica para verificação de todos os itens necessários para uma anestesia ou cirurgia 
segura estão disponíveis. O check list é conduzido pela equipe de enfermagem junto à equipe médica. ${ }^{9}$

Os itens da Cirurgia Segura que são contemplados pela OMS e que devem ser seguidos e para cada item deve ter protocolos assistenciais de segurança implementados: ${ }^{10}$ certo.

- A equipe irá operar o paciente certo e no local

- A equipe usará métodos embasados cientificamente para prevenir danos na administração de anestésicos.

- A equipe reconhecerá efetivamente e se preparará para o risco de perda de via aérea ou de função respiratória.

- A equipe reconhecerá efetivamente e se preparará para o risco de perda sanguínea elevada.

- A equipe evitará a indução de uma reação alérgica ou adversa de drogas (medicamentos) para os quais o risco ao paciente é conhecido.

- A equipe usará métodos conhecidos para minimizar o risco de infecção de sítio cirúrgico.

- A equipe impedirá a retenção inadvertida de instrumentos ou compressas em feridas cirúrgicas.

- A equipe garantirá e identificará com precisão todos os espécimes cirúrgicos.

- A equipe comunicará e trocará informações críticas para a condução segura da cirurgia.

- Hospitais e sistemas de saúde pública estabelecerão vigilância de rotina da capacidade de cirúrgica, dos volumes e resultados.

Nesse programa da OMS para garantir a segurança em cirurgias e contemplar os 10 itens acima, devemos verificar itens essenciais do processo cirúrgico. $\mathrm{O}$ objetivo é garantir que o procedimento seja realizado conforme planejado, atendendo aos " 5 Certos": Paciente (Deve-se instituir e assegurar a Política ou Programa de Identificação do Paciente), procedimento (Deve-se instituir e assegurar a implementação de protocolos, embasamentos científicos e boas práticas), lateralidade (Deve-se instituir e assegurar a prática internacional destinada a cirurgias e outros procedimentos invasivos em que a necessidade da escolha de um lado, por exemplo, um braço direito.

Ela deve ser feita antes do encaminhamento do paciente ao centro cirúrgico), Posicionamento (Seguir metodologias de posicionamento embasadas cientificamente de acordo com cada tipo de cirurgia) e Equipamentos (Assegurar que existe um estoque mínimo de equipamentos, materiais e insumos e que todos estão validados pelo Serviço de Controle de Infecção Hospitalar (SCIH), além de ter um Serviço de Engenharia Clínica que garanta as manutenções preventivas e corretivas desses equipamentos). ${ }^{9}$

Ao paciente a cirurgia segura significa reduzir a um mínimo aceitável o risco de dano desnecessário associado ao cuidado de saúde, porém para que isso ocorra de maneira esperada, é necessária uma série de ações que os profissionais devem atentar para manter a segurança do paciente. O cuidado no pré, trans e pósoperatório determina a qualidade da assistência prestada e a recuperação do cliente submetido ao procedimento cirúrgico. ${ }^{11 ; 12}$

Como também a realização do checklist para o alcance do procedimento correto, pela equipe correta e paciente correto, tendo em vista estabelecer processos seguros na realização da cirurgia. Destacando-se dessa forma as possíveis estratégias e instrumentos utilizados para assegurar o paciente e garantir a qualidade da assistência prestada..$^{11 ; 12}$

Dentro do contexto citado acima fica claro que é amplamente necessário seguir todos os protocolos e manuais sobre cirurgia segura, uma vez que leva a alta diminuição dos fatores de risco e evidencia a melhora no atendimento, no conforto e no cuidado com o paciente.

\section{A relevância do enfermeiro com protocolo de cirurgia segura}

Pela segurança do cliente, busca-se a redução e/ ou atenuação de atos considerados inseguros atrelados ao sistema de assistência à saúde, bem como o emprego das melhores práticas, no intuito de obter os resultados esperados. Ressalta-se ainda que o cuidado seguro ira refletir direta e intimamente a qualidade da assistência prestada à população, que por sua vez, tem adquirido mais ciência de seus direitos, estabelecendo critérios que reflitam suas escolhas e atitudes diante dos serviços de saúde, visando, assim, evitar a ocorrência de eventos adversos. ${ }^{13}$

É atribuição do enfermeiro, portanto, informar o paciente cirúrgico sobre seu problema de saúde, procedimento cirúrgico e, principalmente, como ele poderá participar de sua recuperação pós-operatória, orientando-o no pré e pós-operatório por meio de uma linguagem clara, respeitando seus conhecimentos e sua cultura. É inerente ao papel do enfermeiro a atividade educativa, pois ele permanece diuturnamente com o paciente, podendo promover espaço propício para a orientação e o empoderamento sobre seu autocuidado, fornecendo orientações acerca dos procedimentos e cuidados, além de promover a saúde e prevenir complicações potenciais. ${ }^{14}$

Essa situação torna esse profissional o elemento da equipe de saúde com maior oportunidade para orientar o paciente. Além do mais, é nítida a importância da contribuição da enfermagem no início, durante e após o término da cirurgia, por exemplo, certificando a identidade e o consentimento do cliente, o local e o procedimento; verificando os sinais vitais e mantendo o paciente monitorado e com acesso venoso; eliminando a presença de qualquer risco, como o de perda sanguínea, 
dificuldade das vias aéreas, reações alérgicas e complicações no pós-operatório. ${ }^{13}$

Por outro lado, identificar situações que remetam a possíveis erros na assistência perioperatória ao paciente cirúrgico é o grande desafio do enfermeiro, dentre as quais se destacam as falhas nos equipamentos de anestesia, falta de pessoal capacitado, equipe cirúrgica trabalhando sob pressão, uso das novas tecnologias com pouco conhecimento, entre outras. ${ }^{15}$

Sendo assim, implementar a cultura de segurança nas instituições de saúde, especificamente no Centro Cirúrgico, pode ter associação direta com a diminuição dos eventos adversos e da mortalidade, implicando melhorias na qualidade da assistência à saúde dos pacientes.

\section{Erros e fragilidades que irãopor em risco a segurança do paciente cirúrgico}

As falhas e os eventos adversos que acometem o paciente cirúrgico podem lhe trazer inúmeras consequências, inclusive com agravos permanentes. As falhas podem envolver recursos materiais, tecnológicos e humanos. Em se tratando de recursos humanos, um fator que influencia a segurança do paciente é a concentração no procedimento que está sendo realizado. Estudos apontaram que ocorrem, em média, 60 interrupções ou distrações durante $o$ ato cirúrgico, na maioria das vezes desencadeadas pelo movimento de pessoas na sala. ${ }^{16}$

No ambiente intraoperatório, as fragilidades que põem o paciente em risco são inúmeras, destacando-se a ocorrência de lesões na pele durante o procedimento, nesses casos, destacou-se como fatores de risco para ocorrência desse tipo de lesões o não uso de recursos como protetores para manutenção corpóreo, tempo cirúrgico prolongado e obesidade ou sobrepeso. Outra fragilidade, também evidenciada foi em relação ao estresse entre os profissionais enfermeiros da unidade cirúrgica. ${ }^{15}$

Diante de várias fragilidades e potenciais riscos encontrados e apesar da recomendação do uso do protocolo de Cirurgia Segura pela OMS, diversas instituições ainda não aderiu de maneira significativa à realização do checklist na conferência das informações de jejum, tricotomia, ausência de esmaltes e adornos, identificação do paciente e do sítio operatório, disponibilidade de sangue, verificação da lateralidade, apresentação da equipe e contagem de materiais na sala. ${ }^{15 ; 16}$

Dessa forma, faz-se necessário enfatizar cada vez mais o caráter assistencial e clínico do enfermeiro, para que esse profissional possa executar atividades próximas ao paciente, reconhecendo assim as necessidades pertinentes a ele e podendo promover um cuidado individualizado e integral ao paciente cirúrgico, o que interfere também no reconhecimento e na valorização da profissão.

\section{Conclusão}

Com base nos resultados obtidos através desse estudo fica evidenciado que o protocolo de cirurgia segura é uma importante fonte do cuidado imposto, para que através dele se possa garantir de uma assistência integral, mesmo que não ocorra de forma ideal, deverão suprir as necessidades básicas de segurança do procedimento cirúrgico e do paciente.

Todavia essa assistência terá sua qualidade alterada, principalmente se ele for caracterizado como algo não necessário pelos profissionais, mudando completamente sua rotina, surgindo desta maneira uma das principais queixas do paciente que será submetido à cirurgia, que simboliza diversos pontos negativos, resultando a partir daí uma serie de fatores emocionais e físicos, que poderá levar ao ato cirúrgico sem sucesso.

Desta maneira conclui-se a importância de seguir o protocolo e da equipe de saúde realizar todo o processo de maneira adequada, principalmente dos enfermeiros, que tem uma função primordial para garantir a eficácia do check list e de todo o cuidado com o paciente mediante os diversos fatores negativos que vão aparecer durante essa enorme jornada, mediante ao desgaste e despreparo da maioria para com o papel.

\section{Referências}

1. Alberti GF, Ribeiro MG, Velasque ACI, Salbego C, Dornelles CS. A Prática de Enfermagem em Centro Cirúrgico: A utilização do índice de Ladre-te e Kroulik para a avaliação do paciente em sala de recuperação pós-anestesica. Florianópolis Jul./Set. 2016. Disponível em: http://www. urisantiago.br/saenfermagem/anais/2010/09\%20A\%20PRATICA\%20 DE $\% 20$ ENFERMAGEM $\% 20$ EM $\% 20$ CENTRO $\% 20$ CIRURGICO $\% 20$ A\%20UTILIZACAO $\% 20 . p d f$

2. AMATO, Instituto de Medicina Avançada, São Paulo, 2014. Disponível em: https:/www.amato.com.br/content/como-funciona-equipena-sala-de-cirurgia

3. Arcêncio L, Marilize MD, Bortolin BS. Cuidados pré e pós-operatórios em cirurgia cardiotorácica: uma abordagem fisioterapêutica. Rev Bras Cir Cardiovasc. Ribeirão Preto, São Paulo. 2008; 23(3): 400-410. Disponível em: http://www.scielo.br/pdf/rbccv/v23n3/v23n3a19

4. Brasil. Ministério da Saúde. Documento de referência para o Programa Nacional de Segurança do Paciente. 2014. Disponível em: http:// portalms.saude.gov.br/acoes-e-programas/programa-nacional-de-segurancado-paciente-pnsp/implantacao-do-programa-nacional-de-seguranca-dopaciente

5. Camanho GL. Cirurgia Segura para Todos. Rev. bras. ortop. São Paulo, 2014 Dez.; 49(6). Disponível em: http://dx.doi.org/10.1016/j. rboe. 2014.09 .00

6. Carvalho ARS, Matsuda LM. Investigando as orientações oferecidas ao paciente em pós-operatório de revascularização miocárdica. Rev Eletrônica Enferm. 2008;10(2):504-12. Disponível em: http://www.revenf. bvs.br/scielo.php?script=sci arttext\&pid=S1518-19442008000200020\&lng $=\mathrm{pt} \& \mathrm{nrm}=\mathrm{iso} \& \mathrm{tlng}=\mathrm{pt}$

7. Grigoleto A, Gimenes F, Avelar M. Segurança do cliente e as ações frente ao procedimento cirúrgico. Rev. Eletr. Enf. 2011; 13(2) Disponível em: http://dx.doi.org/10.5216/ree.v13i2.10326 
8. Lima AM, Sousa CS, Cunha ALSM. Segurança do paciente e montagem de sala operatória: estudo de reflexão. Rev enferm UFPE. 2013; 7(1): 289-94. Disponível em: file://C:/Users/Iago\%20Freitas/ Downloads/10232-20427-1-PB\%20(1).pdf

9. Minayo MCS. O desafio do Conhecimento. 10. ed. São Paulo: HUCITEC, 2009. Disponível em: http://www.ufrgs.br/cursopgdr/ downloadsSerie/derad005.pdf

10. Rezende JM. À Sombra do Plátano: Crônicas de História de Medicina. São Paulo: Unifesp, 2009. Disponível em: http://books.scielo.org/id/8kf92/ pdf/rezende-9788561673635.pdf

11. Richardson RJ. Pesquisa social: métodos e técnicas. São Paulo: Atlas, 2009. Disponível em: http://www.ufrgs.br/cursopgdr/downloadsSerie/ derad005.pdf

12. Santos J, Henckmeier L, Benedet SA. O impacto da orientação préoperatória na recuperação do paciente cirúrgico. Enferm. Foco. 2011; 2(3): 184-7. Disponível em: http://pesquisa.bvsalud.org/portal/resource/pt/bde24907

13. Silva A, Silva FA. Equipe de enfermagem em cirurgia segura: desafios para adesão ao protocolo. Rev. enfer. UFPI; 6(2): 23-29, abr.-jun.2017. Disponível em: http://pesquisa.bvsalud.org/portal/resource/pt/bde-31983

14. Silva DG. Grupos como possibilidades para desenvolver educação em saúde. Texto Contexto Enferm. 2013;12(1):97-103. Disponível em: http://ojs. unifor.br/index.php/RBPS/article/view/4410document/view/ztkzfdocument/ view/2njhg

15. Souza RM, Araújo MG, Verrisímo RC, Comasseto I, Ferreira FA. Aplicabilidade do check list de cirurgia segura em Centro Cirúrgicos hospitalares. Rev. SOBECC 2016 out.-dez.; 21(4): 192-197. Disponível em: http://pesquisa.bvsalud.org/portal/resource/pt/biblio-831532

16. Tiossi M, Marin A. Centro Cirúrgico: É possível humanizar? REVISTA UNINGÁ, [S.1.], 2017out.;2(1). Disponível em: http://revista. uninga.br/index.php/uninga/article/view/359. 